\title{
COVID-19 and Acute Cardiovascular Diseases in Bangladesh- A Time Tested Medical Emergency to deal with
}

\author{
AHM Waliul Islam, Shams Munwar, AQM Reza, Shahab Uddin Talukder, Azfar H Bhuiyan, \\ Tamzeed Ahmed, Md Atahar Ali, Kazi Atikur Rahman, Md. Shamsul Alam \\ Interventional Cardiology Department, Evercare Hospital Dhaka
}

\begin{abstract}
:
Key words:

COVID-19,

Covid-19 pandemic has affected millions of patients, killed more than half a million, and poses major health threat globally. There is high demand for clinical resources and bed space for COVID19 patients. Admission of patient of any cardiac emergency in a common block of hospital, country STEMI, Primary PCI, ACE2 like Bangladesh, may exposes the risk of infection among non-COVID patient. Patient with COVID19 and pre-existing cardiovascular diseases (CVD) have increased risk of further aggravation and death. COVID-19 infection is usually associated with multiple direct and indirect cardiovascular complications, such as acute myocardial injury, myocarditis, arrhythmias, heart failure and venous thromboembolism. Health care worker may pose themselves in vulnerability as they become host or vectors of virus transmission. Defeating COVID-19 and managing acute cardiovascular emergencies in COVID-19 era exposes non-COVID-19 patient, may need dramatic reshaping of care delivery, novel approaches to patient's management and large-scale reallocation of resources.
\end{abstract}

(Cardiovasc. j. 2020; 13(1): 75-80)

Coronavirus disease (COVID-19) caused by the novel severe acute respiratory syndrome corionavirus-2 (SARS-CoV-2) is a major threat to global health including Bangladesh. Since, its recognition in Wuhan, China in December 2019, COVID-19, has rapidly spread throughout the world and become a pandemic. ${ }^{1}$ It has been documented more than $80 \%$ of affected people remain asymptomatic and can be treated symptomatically at home isolation, with recommended mandatory uses of Mask, safe distancing of about 6 feet and 20 seconds hand washing before or after touching any surfaces which might have contaminated with corona virus. Only, $20 \%$ of Covid-19 positive patient may need hospitalization and 3\%-4\% may need ICU care with or without ventilation support. High flow Nasal cannula has proven effective alternative to ventilation.

Bangladesh, a densely populated country over 17 crore people. Many are living below standard of basic needs and lives on daily income. Within the available resources, and very low number COVID19 test, already it is difficult to assess the exact scenario of number of affected people, disease spread, and death from it.

Preparedness to dealt it at very early stage and effective home or institutional isolation, lack of concerned institutional common consensus in preventing the spread and treating facilities with high flow nasal cannula (HFNC) and ICU back up with trained manpower, put us vulnerable to Covid19 infection, with subsequent life-threatening complications and possible long duration.

It has been well documented that in the west, old age people, male sex, with multiple comorbid are in great risk with highest mortality in this subset of patient. Among those, who had COVID-19 diagnosis by RT-PCR, more than 14 million peoples infected by COVID-19 and more than 6.2 lac died.

Address of Correspondence: Prof. Dr. AHM Waliul Islam, Dept. of Interventional cardiology, Evercare Hospitals Dhaka, Bangladesh. E-mail: waliul.islam@evercarebd.com

- 2020 authors; licensed and published by International Society of Cardiovascular Ultrasound, Bangladesh Chapter and Bangladesh Society of Geriatric Cardiology. This is an Open Access article distributed under the terms of the CC BY NC 4.0 (https://creativecommons.org/licenses/by-nc/4.0) 
Many remain undiagnosed and died at home with the symptoms of corona.

Currently, there is no evidence from randomized clinical trials (RCTs) that any potential therapy improves outcomes in patients with either suspected or confirmed COVID-19. There is no clinical trial data supporting any prophylactic therapy as well. ${ }^{2}$

In Bangladesh, the number of acute cardiovascular condition dramatically fall in most of the hospitals. The infection caused by severe acute respiratory syndrome coronavirus-2, COVID-19, can result in myocardial injury, heart failure and arrhythmias. Therapies for COVID-19 has many adverse cardiac effects. In Wuhan, two recent papers found higher troponin level in $27.8 \%$ and $19.7 \%$ of patients, and were older age, had higher rates of comorbid conditions including hypertension, coronary artery disease, history of cardiomyopathy, chronic obstructive pulmonary disease. ${ }^{3-4}$

ACC/AHA/ESC published guidelines in treating ST segment Elevated Myocardial Infarction (STEMI) patients along with other life-threatening cardiac conditions. For, acute ST elevated myocardial infarction, they recommended initially, pharmacoinvasive therapy. Where, Patient will be thrombolysed and later, to Rescue or elective PCI. Some recommended for Primary PCI if there is Corvid-19 dedicated Cardiac catheterization lab available. ${ }^{5-6}$

In Bangladesh, most of the center has only one Cath lab and it's not possible to treat STEMI patient in this global pandemic, where chances of possible infection to non-COVID-19 patient. Since, like west, we do not have separate block or facilities to treat only COVID-19 patient. Therefore, we started at our center pharmaco-invasive therapy, initially thrombolysis by STK or TNK in grey zone of our CCU, send COVID-19 test, if positive then to treat him conservatively and wait till his COVID-19 become negative, to perform coronary angiogram with or without required intervention or CABG.

It has already shown in various research on modifiable risk factors that potentially increased susceptibility to infection or worst outcomes among patient with cardiac disease, CKD, Diabetes Mellitus. ${ }^{7-8}$ It has been well established that the SARS-Cov-2, a single stranded RNA-enveloped virus, targets cell through the Spike protein of COVID-19 binds ACE2 receptors in Lung Alveoli and then enter into cell, where replicate to several thousand virus. ${ }^{9}$ Also, through its binding affinity with ACE2 receptor, COVID-19 binds heart, kidney, and vascular system. Several has hypothesized, that Angiotensin Converting Enzymes inhibitor (ACEIs)/ angiotensin receptor blockers (ARBs) may make patients more susceptible to COVID-19 and to worse outcomes through upregulation of the functional receptor of the virus ACE2.10-12 Therefore, whether to discontinue or add the drugs, several has emphasized that the available data do not support discontinuation or addition of ACEI/ ARB. ${ }^{13-14}$ In a high-risk population of older patients with cardiovascular disease, randomization to ramipril had no impact on the incidence or severity of COVID-19 and support the maintenance of RAAS inhibitor treatment during COVID-19 crisis. ${ }^{15}$

These viral lifecycle steps provide potential targets for drug therapy from binding to spike proteins to cell entry, synthesizes RNA via RNA dependent RNA polymerase and finally release of viral particles. ${ }^{16}$ At present, absence of proven therapy for SARS-CoV-2, the cornerstone of care for patient COVID-19 remains supportive care, ranging from symptomatic OPD management to full intensive care support. However, 3 adjunctive therapies that warrant special mention are corticosteroids, anticytokine or immunomodulatory agent, and immunoglobulin therapy. The rationale for the use of corticosteroids is to decrease the host inflammatory response in the lungs, which may lead to acute lung injury and acute respiratory distress syndrome (ARDS). ${ }^{17}$ Although direct evidence of corticosteroids for Covid-19 is limited, delayed viral clearance and increased risk of secondary infection may not rule out. Study on 201 Chinese Covid-19 patients, treatment with methylprednisolone was associated with decreased risk of death $23 / 50$ (46\%) with steroids vs $21 / 34$ (62\%) without; HR, 0.38 (95\% CI, 0.20-0.72). ${ }^{18}$ Cytokine storm might mediate lung and other organ damage and IL-6 appears to be a key driver of this dysregulated inflammation in Covid-19 cases. Tocilizumab, a monoclonal antibody IL-6 receptor antagonist associated with clinical improvements in $91 \%$ patients. ${ }^{19}$

Another potential adjunctive therapy for Covid-19 is the use of convalescent plasma or hyperimmune 
immunoglobulins. ${ }^{20}$ The rationale for this treatment is that antibodies from recovered patients may help with both free virus and infected cell immune clearance. In theory, the benefit of this therapy would accrue primarily within the first 7-10 days of infection, when viremia is at its peak and primary immune response has not yet occurred.

COVID-19 has dramatically altered the way of life for billions around the globe. Concern has directed at how to limit the spread of virus, pressure on whole healthcare system, and overall increasing mortality and morbidity from this devastating pandemic. While in many places of world including our Bangladesh, acute cardiovascular disorders have fell but disappeared from many of the hospital emergency departments and hospital words.

Recently, Bhatt et al, has published in JACC, showed that in USA, $43 \%$ reduction hospital admission for patient with acute coronary syndrome or acute heart failure, since community spread of SARS-CoV-2. Also, total length of hospital stay was 1.2 days shorter and greater rates of inhospital death than patients in the preceding 15 months. $^{21}$

We, all knew that COVID-19 has revealed a large systemic inflammatory response, with elevations in erythrocyte sedimentation rate, C-Reactive protein, Ferritin, D-dimer and Interleukin-6 levels. Such robust inflammation would be expected to drive increases in plaque destabilization and atherothrombotic events with resultant acute coronary syndrome and cerebrovascular events. ${ }^{22}$

In managing COVID-19, and to prevent its spread, governmental agencies, medical societies and public health organizations stressing the importance of staying home. Thus, like west and developed world, to avoid unnecessarily expose to COVID-19. Patient in Bangladesh, also remain home bound, until the symptoms of acute cardiac emergencies become unbearable.

In the global pandemic COVID-19 era, it has been reported in other parts of world, that there are almost 40\% reductions in admission for ACS and catheterization lab activation for STEMI patients. ${ }^{23-24}$ The large number of COVID-19 infected patients requiring care may affect the optimal treatment require for those with acute cardiac conditions. Patient with known cardiac diseases, if infected with or chance to expose COVID-19, might have higher risk of adverse outcomes, with prolong hospital and intensive care stay, as infection itself is associated with or can cause myocardial injury with CV complications like heart failure and arrhythmia. ${ }^{25-26}$

Early identification and isolation of cardiovascular patients with COVID-19 symptoms from other as is critically important. It is prudent to advise all cardiovascular patients of increased risk and isolation and self-distancing. Acute viral infections have multiple short terms effects on CV system like ACS, Myocarditis or worsening LV dysfunction leading to Left Ventricular Failure (LVF) and arrhythmias, with possible shock.

Myocardial injury in patients with COVID-19 could be due to plaque rupture, cytokine storm, hypoxic injury, coronary spasm, microthrombi, or direct endothelial or vascular injury. ${ }^{27}$ Bangalore et al, ${ }^{28}$ has demonstrated that a total of 18 COVID patients with 10 has STEMI at presentation and 8 patient developed STEMI during hospitalization. Total, 9 (50\%) patients had CAG, 6 (67\%) had obstructive disease and 5(56\%) underwent PCI (one after administration of fibrinolytic agents). 10(56\%) patients had noncoronary myocardial injury. A total 13(72\%) died (4 STEMI and 9 noncoronary MI). Ddimer and Trop-I were higher. These findings indicating that majority of STEMI patient with COVID-19, may have normal coronaries on CAG and MI may be due to the consequences of viral infection as described above.

Primary Percutaneous Coronary Intervention (pPCI) is a common modality of treating patient with acute STEMI with better myocardial salvage at our center. Since the first detection of COVID19 in Bangladeshi patient on March 8, 2020, millions have affected, several thousands died, and several are in critical condition admitted in hospital. Number of pPCI in STEMI patient has dramatically fall in our center, which is open for PPCI round the clock 7/24. The reason, possibly, patients and relatives are afraid of COVID-19 infection, who were known to other comorbidities.

We have set protocol of not to go for primary PCI for patients presenting with STEMI in our Emergency Department. Since, we do not know the background if he is already infected with 
COVID-19 or not. Thus, as a part of pharmacoinvasive therapy, first to thrombolyse either by STK or Tenecteplase (TNK) and manage in CCU. Once patient settled, and treat conservatively and perform CAG, and PCI after becoming corona Negative. For those, who were remain symptomatic with ongoing angina, do CAG and intervention, as per requirement with suspecting the [patient as COVID-19 positive, since RT-PCR is $60-70 \%$ sensitive and negative does not rule out the infection, may turn positive later on.

Very recently, in New York Metropolitan area assess the epicenter of the COVID-19 pandemic.

Ambulatory care was dramatically curtailed, emergency departments (ED) overwhelmed, and Patients were advised to stay home. ${ }^{29}$ A reduction in patients presenting with acute coronary Syndromes (ACS) during the crisis was reported. ${ }^{30}$ If patients were staying home with ACS, a delay in presentation with worsening symptoms or a rise in out-of-hospital sudden cardiac arrest (OHCA) would be expected. A 58\% increase in OHCA early in the COVID-19 outbreak was reported in Italy. ${ }^{31}$

Researcher has found that COVID-19 patients, presenting with STEMI had higher levels of Troponin T, lower lymphocyte count, elevated Ddimer and C-reactive protein. There was also found higher rates of multivessel thrombosis, higher uses of GP IIb/IIIa inhibitors and thrombus aspiration. Myocardial blush and left ventricular function were lower in this group of patients, in association with longer ICU admission. Thus, recommended more aggressive anti-thrombotic therapy in STEMI patients with COVID-19. ${ }^{32}$

ACC/SCAI has recommendation to balance of staff exposure and patients benefit and to preserve hospital bed. Thus, to avoid primary PCI in STEMI cases, whom hospital stay is $>1$ to 2 days and who may need ICU admission. Fibrinolysis can be considered an option for a relatively stable patients with STEMI with active COVID. ${ }^{33}$

In Bangladesh, most of the cardiac Cath lab is not designed for infection Isolations and most of the hospital are in one building block, with no available isolated wing where only COVID-19 patient can be treated. Still we have not achieved peak and we do not have any definitive projected data, on how many days, we have to fight with this global pandemic. In any circumstances, if it is prolonged, then we may need to find out way to do primary PCI with precaution both for operator and patients with full protective gear, and fumigation of Cath lab after each procedure. So, non-COVID patient can be treated, later.

Thrombotic complications seem to emerge as an important issue in patients infected with COVID19. Preliminary reports on COVID-19 patients, clinical and laboratory findings include thrombocytopenia, elevated D-dimer. ${ }^{34}$ Emerging data support that patients infected with this virus are in increased risk of disseminated intravascular coagulation. Increased D-diner, and fibrin degradation product levels and prolonged prothrombin time have been associated with poor prognosis. ${ }^{35}$ To prevent thrombo-embolic manifestation, patients with COVID-19 infections are being treated with low-molecular heparin or unfractionated heparin, dabigatran, rivaroxaban.

In addition, lifestyle modification, including walking or light exercise while staying at home, stop smoking and control of Diabetes Mellitus, Blood pressure, avoidance of excess carbohydrate specially rice, sugar, potato. Protein restriction for patient with chronic renal diseases is mandated. Fluid restriction for LVF patient with low Ejection Fraction, should maintained strictly. Extra table salt should be avoided for high blood pressure patient.

\section{Conclusion:}

Spread of COVID-19 in our society can overwhelm health systems. The sickest patients might be older in age, with cardiovascular Co-morbidities along with acute kidney Injury, Diabetes Mellitus and chronic Bronchial Asthma. Many of the known cardiovascular patients are in potential threat to COVID-19, already had PCI, CABG or Pacemaker, ICD or CRT-D. The mainstay treatment of COVID19 infection is supportive therapy. We recommend avoiding unnecessary hospital contact or expose to life threatening COVID-19 infections. Already diagnosed cardiac patient can be follow-up on regular telehealth and can be triaged for emergency needs of cardiac catheterization and EP study.

In developing country like Bangladesh, with an already frail health system due to economic 
problem, lack of integrity among the various health care provider authority, a lot of irregularities, the COVID-19 is more challenging to dealt it where many of the front fighter specialist Doctors lost their lives and many are suffering. There is urgent time demand needs in the formulation of efficient protocols that can accommodate several medical, and socioeconomic challenges including any cardiac emergencies, especially acute STEMI and LVF.

\section{Conflict of Interest - None.}

\section{References:}

1. Zhu N, Zhang D, Wang W, et al. China Novel Coronavirus Investigating and research Team. A novel coronavirus from patients with pneumonia in China, 2019. N Eng. J Med 2020(8): 727-7333. doi:10,1056/ NEJMoa2001017

2. Sanders JM, Mongue LM, Jodlowski TZ, et al. Pharmacologic treatment for coronavirus disease Covid19. JAMA 2020;323(18):1824-1836

3. Guo T, Fan Y, Chen M, et al, cardiovascular implications of fatal outcomes of patients with coronavirus disease 2019 (COVID-19). JAMA Cardiol 2020, http//doi.org/ 10.1001/jamacardiol.2020.1017

4. Shi S, Qin M, Shen B, et al. Association of cardiac injury with mortality in hospitalized patients with COVID-19 in Wuhan, China. JAMA Cardiol 2020, http//doi.org/ 10.1001/jamacardio.2020.0950

5. Mahmud E, Dauerman HL, Welt F, et al. Management of Acute Myocardial Infarction During the COVID During the COVID During the COVID During the COVID-19 Pandemic. A Consensus Statement from the Society for Cardiovascular Angiography and Interventions (SCAI), American College of Cardiology (ACC), and the American College of Emergency Physicians (ACEP). J Am Coll Cardiol 2020. doi: 10.1016/ j.jacc.2020.04.039

6. The European Society for Cardiology. ESC Guidance for the Diagnosis and Management of CV Disease during the COVID-19 Pandemic. https:// www.escardio.org/education/COVID-19-and-Cardiology /ESCCOVID-19-Guidance.

7. Diaz JH. Hypothesis: angiotensin converting enzyme inhibitors and angiotensin receptor blockers may increase the risk of severe covid-19. J Travel Med 2020.27(3): ta041. doi:1001093/jtm/taaa041

8. Fang L, Karakiulakis G, Roth M. Are patients with hypertension and diabetes mellitus at increased risk for Covid-19 infection? Lancet Respir Med 2020;8(4): e21.doi:10.1016/S2213-2600(20)30116.8Hoffman M, Kleine-Weber H, Schroeder S et al. SARS-Cov-2 cell entry depends on ACE2 and TMPRSS2 and is blocked by clinically proven protease inhibitor. Cell 2020:181(2):271-280.e8. doi: 10.1016/j.cell.2020.2.05

9. Esler M, Esler D. Can Angiotensin receptor blocking drugs perhaps be harmful in Covid 19 pandemic. $J$ Hypertension 2020: 38(5):781- 782

10. Mehta N, Kalra A, Nowacki AS, et al. Association of use of angiotensin converting enzymes inhibitors and angiotensin ii receptor blockers with testing positive for coronavirus disease 2019(Covid-19). . Published online May 5, 2020.doi:10.1001/jamacardio.2020.1855

11. Reynolds HR, Adhikari S, Pulgarin C, et al. Reninangiotensin-aldosterone system inhibitors and risk of Covid -19. N Engl J Med. published online May 1, 2020.doi:10.1056/NEJMoa2008975

12. Bozkurt B, Kovacs R, Harrington R, et Al. HFSA/ACC/ AHA statement addresses concerns reusing RAAS antagonist in Covid-19. Published 2020. Accessed May 2,2020

13. European Society of Cardiology. Position statement of the ESC council on hypertension on ACE-inhibitors and angiotensin receptor blockers. Published March 13,2020. Accessed June 2020

14. Vaduganathjan M, Vardeny O, Michel T, et al. Reninangiotensin-aldosterone inhibitors in patients with Covid-19. N Engl J Med 2020.382(17): 16531659.doi:10.1056/NEJMsr2005/60

15. Amat-Santos IJ, Santos-Martinez S, Lopez-otero D, et al. Ramipril in High Risk Patients with COVID-19. $J$ Am Coll Cardiol 2020; 76:268-276.

16. Chen Y, Liu Q, Guo D. Emerging coronavirus: genome structure, replication and pathogenesis. J Med Virol 2020;92(4):418-423. Doi:10.102/jmv25681

17. Russel CD, Miller JE, Baillie JK, et al. Clinical Evidence does not support corticosteroids treatment for 2019 nCoV lung injury. Lancet 2020 395(10223):473-475. Doi:10.1016/S0140+6736*(20)30317

18. Wu C, Chen X, Cai Y, et al. Risk factors associated with acute respiratory distress syndrome and death in patients with coronavirus disease 2019 pneumonia in Wuhan, China. JAMA Intern Med. Pub online on March 31, 2020

19. Xu X, Han M, Li T, et al Effective treatment of severe Covid-19 patients with Tocilizumab. Chinaxiv. Preprint post March 2,2020. Doi;10.12074/202003.00026

20. Zhou F, Yu T, Du R, et al. Clinical course and risk factors for mortality of adult inpatients with Covid-19 in Wuhan, China. A retrospective cohort study. Lancet 2020;395(10229); 1054-1062.doi.10.1016//S01406736(20)30566-3

21. Bhatt AS, Moscone A, McElrath Fewer hospitalizations for acute cardiovascular conditions during COVID-19 pandemic. J Am Coll Cardiol 2020; 76:280-288.

22. Giamarrellos-Bouboulis EJ, Netea MG, Rovina N, et al. Complex immune dysregulation in COVID-19 patients with severe respiratory failure. Cell Host Microbe 2020; 27:992-1000e3 
23. Garcia S, Albaghdadi MS, Meraj PM, Schmidt C, Garberich R, Jaffer FA et al. Reduction in ST-Segment Elevation Cardiac Catheterization Laboratory Activations in the United States During COVID-19 Pandemic. 2020 Jun 9;75(22):2871-2872. doi: 10.1016/ j.jacc.2020.04.011. Epub 2020 Apr 10.

24. De Filippo O, D’Ascenzo F. Angelinin F, et al. Reduced rate of hospital admissions for ACS during covid-19 outbreak in Northern Italy. N Engl J Med 2020 Apr 28 (E-pub ahead of print)

25. Li B, Yang J, Zhao F, et al. Prevalence and impact of cardiovascular metabolic diseases on COVID-19 in China. Clin Res Cardiol 2020 Mar 11 (E-pub ahead of print)

26. Zheng YY, Ma YT, Zhang JY, et al. COVID-19 and the cardiovascular system. Nat Rev Cardiol 2020 Mar 5 (Epub ahead of print)

27. Tavazzui G, Pellegrini C, Maurelli M, et al. Myocardial localization of coronavirus in COVID-19 cardiogenic shock. Eur J Heart Fail 2020 April 10 (Epub ahead of print)

28. Bangalore S, Sharma AS, Slotwiner A, et al. STSegment Elevation in patients with COVID-19- A case Series. N Engl J Med doi.10.1056/NEJMc2009020

29. Center for Disease Control and Prevention. (2020). Interim Guidance for Implementing Home Care of People Not Requiring Hospitalization for Coronavirus
Disease 2019 (COVID-19). Retrieved from cdc.gov/ COVID19.

30. Mountantonakis SE, Saleh M, et al. Out of Hospital cardiac arrest and acute coronary syndrome hospitalizations during the Covid-19 surge. J Am Coll Cardiol (2020). doi: 10.1016/j.jacc.2020.07.021.

31. Choudry FA, Hamshere SM, Rathod KS, et al. High Thrombus Burden in Patients with COVID-19 Presenting with ST-Elevation Myocardial Infarction. $J$ Am Coll Cardiol (2020). doi: 10.1016/j.jacc.2020.07.022.

32. Welt FGP, Shah PB, Aronow HD, et al. Catheterization laboratory considerations during the coronavirus (COVID-19) Pandemic. J Am Coll Cardiol; doi.10.1016/ j.jacc.2020.03.021

33. Bainey KR, Bates ER, Armstrong PW, et al. STSegment-Elevation Myocardial Infarction Care and COVID-19 The Value Proposition of Fibrinolytic Therapy and the Pharmcoinvasive Strategy. Circ Cardiovasc Qual Outcomes. 2020;13: e006834. DOI: 10.1161/ CIRCOUTCOMES.120.006834

34. Guan WJ, NI ZY, Hu Y, et al. China Medical treatment expert group for Covid-19. Clinical characteristics of coronavirus disease 2019 in China. N Engl J Med 2020. Doi;10.1056/NEJMoa2002032

35. Tang N, Li X, Wang Z, et al. Abnormal coagulation parameters are associated with poor prognosis in patient's novel coronavirus pneumonia. J Thromb Hemostat JTH 2020; 18(4):844-847. 